\title{
PROTOCOLO ASSISTENCIAL OBSTÉTRICO: ORIENTAÇÕES PARAA SEGURANÇA E HUMANIZAÇÃO DA PARTURIENTE
}

\section{CARE PROTOCOLS: SAFETY AND HUMANIZATION GUIDELINES FOR PARTURIENT WOMEN}

\author{
Anaíle Pires dos Santos ${ }^{1}$ \\ Maristela Conceição Lago Lima² \\ Camila Torres da Paz \\ Rita de Cássia Calfa Vieira Gramacho ${ }^{4}$ \\ Beatriz Guimarães Gentil Fraga ${ }^{5}$
}

\begin{abstract}
Os protocolos permitem que os enfermeiros obstétricos prestem assistência mais segura e humanizada em todo o ciclo gravídico-puerperal. Ter um parto seguro e humanizado envolve um conjunto de práticas, que se realizadas corretamente, irão prevenir a morbimortalidade materna e perinatal. Além disso, os protocolos, que são embasados pela legislação e seguem uma conduta humanizada, proporcionam à mulher o direito à privacidade e à autonomia durante o seu parto. Assim, este estudo tem como objetivo relacionar os protocolos assistenciais utilizados pelos enfermeiros obstétricos, para promoção da segurança e a humanização do cuidado à parturiente, no âmbito do Sistema Único de Saúde. Trata-se de um estudo de revisão integrativa da literatura, de natureza descritiva, com abordagem qualitativa. Os dados foram coletados no período de janeiro a maio de 2018 e as discussões feitas a partir dos pontos de convergência e divergência dos autores. Logo em seguida foram organizadas com base em três categorias de análise. Encontrou-se que os protocolos buscam reorganizar a assistência, bem como garantir a sua qualidade desde o pré-natal até o puerpério, e minimizar os procedimentos desnecessários. Conclui-se que os protocolos fortalecem a autonomia dos enfermeiros obstétricos, exaltam o reconhecimento de suas competências e, principalmente, asseguram os direitos da parturiente.
\end{abstract}

Palavras-chave: Boas Práticas. Humanização do Parto. Protocolos Assistenciais.

The protocols allow obstetric nurses to provide safer and more humane care in any pregnancy-puerperal cycle. Having a safe and humanized delivery involves a set of practices that if correctly performed will prevent maternal and perinatal morbidity and mortality. In addition, protocols that are based on legislation and follow a humanized behavior provide women with the right to privacy and autonomy during delivery. Thus, this study aims to relate the assistance protocols used by obstetrical nurses to promote safety and the humanization of parturient care within the framework of the Unified Health System. In this integrative literature review data were collected from January to May of 2018 and the discussion is based on the points of convergence and divergence of the authors. Data were then organized based on three categories of analysis. It was found that the protocols seek to reorganize care, as well as ensure quality from prenatal to puerperium, while minimizing unnecessary procedures. It is concluded that the protocols strengthen the autonomy of obstetrical nurses, recognize their competence and, mainly, assure the rights of the parturient.

Keywords: Good Habits. Humanization of Childbirth. Assistance Protocols.

\footnotetext{
1Pós-graduanda do Curso de Especialização em Enfermagem Obstétrica da Escola Bahiana de Medicina e Saúde Pública (EBMSP). E-mail: anaile_pires@yahoo.com.br

2Pós-graduanda do Curso de Especialização em Enfermagem Obstétrica da Escola Bahiana de Medicina e Saúde Pública (EBMSP). E-mail: stela.lagolima@hotmail.com

${ }^{3}$ Enfermeira Obstetra; Mestra em Desenvolvimento Regional e Meio Ambiente; Docente da graduação em enfermagem da Faculdade Maria Milza (FAMAM) e da especialização em enfermagem obstétrica da Enfermagem Obstétrica da Escola Bahiana de Medicina e Saúde Pública (EBMSP). E-mail: camilatorrespaz@gmail.com

${ }^{4}$ Enfermeira Obstetra; Docente do Centro Universitário Jorge Amado (UNIJORGE); Membro da ABENFO Nacional; Diretora da Maternidade Tsylla Balbino; Coordenadora do Curso da Pós-Graduação em Enfermagem Obstétrica da Escola Bahiana de Medicina e Saúde Pública (EBMSP).E-mail: ritacalfa@hormail.com

${ }^{5}$ Acadêmica em Bacharelado em Enfermagem pela Faculdade Maria Milza (FAMAM). E-mail: fragabia@hotmail.com
} 


\section{INTRODUÇÃO}

Historicamente, o processo de nascimento é considerado um evento natural, sendo um momento intrínseco e particular vivenciado por mulheres de diferentes culturas, além de ser uma experiência compartilhada entre seus familiares desde as primeiras civilizações (SANTOS, MELO, CRUZ, 2015; POSSATI, 2017). Estas mulheres eram assistidas por parteiras, que eram reconhecidas por sua grande experiência e prática, mesmo não possuindo conhecimento científico.

No entanto, o declínio da profissão de parteiras começou a ocorrer em meados do século XX, na década de 40, com a incorporação de novas tecnologias e intensificação da hospitalização do parto. Assim, as mulheres que antes eram as protagonistas do processo e tinham seus filhos em seus domicílios, passaram a ser atendidas em instituições de saúde que incorporaram a medicalização, sendo acompanhadas por profissionais, que acabaram se tornando os principais protagonistas (BRASIL, 2001; GOMES, 2014; GONÇALVES, 2011; MALHEIROS, 2012).

O avanço da obstetrícia trouxe muitos benefícios no que diz respeito aos indicadores de morbimortalidade materna e perinatal. Porém, tais avanços acabaram por expor demasiadamente as mulheres e os recém-nascidos a intervenções, muitas vezes desnecessárias (BRASIL, 2017). Este fato é contrário ao que é preconizado mundialmente para as práticas obstétricas (GONÇALVES, 2011).

Em 2011, o Ministério da Saúde (MS) criou o Projeto Rede Cegonha no âmbito do Sistema Único de Saúde (SUS), embasado nos princípios da humanização e assistência à mulher, com atenção humanizada à gravidez, ao parto e puerpério, ao recém-nascido $(R N)$ e à criança, tendo entre seus objetivos a redução da morbimortalidade materna e perinatal (BRASIL, 2011).

Um grande destaque deve ser dado também ao Programa de Humanização no Pré-Natal e Nascimento (PHPN), criado em 2000, com o intuito de garantir o direito de escolha das mulheres, estimular os profissionais à prática de um atendimento obstétrico integral e de qualidade, bem como reduzir as intervenções durante o parto (MALHEIROS, 2012).

Para que a humanização seja efetivada em ações de atenção à saúde, a Organização Mundial da Saúde (OMS) recomenda uma maior participação dos enfermeiros obstétricos na atenção ao parto, tomando como referência o fato de que estes profissionais, durante sua formação, são orientados para o cuidado e não para a intervenção, e respeitam o parto como sendo algo fisiológico da mulher, além de possuírem habilidades e competências que garantem uma assistência integral à mulher, além de colaborarem com a redução dos índices de morbimortalidade materna e perinatal (REIS, 2015; RABELO; OLIVEIRA,2010).

Ao longo dos anos muito tem se feito em busca de um parto mais seguro e de redução dos índices de mortalidade e morbidade. Houve, nesse sentido, um crescimento no número de diretrizes, normas e protocolos implementados com a finalidade de garantir uma melhor assistência obstétrica e minimizar as práticas intervencionistas. No entanto, apesar do empenho, os indicadores não têm se modificado tanto quanto desejado (REIS, 2015).

Dessa forma, o trabalho teve como objetivo relacionar os protocolos assistenciais utilizados pelos enfermeiros obstétricos para a promoção da segurança e a humanização do cuidado à parturiente no âmbito do Sistema Único de Saúde.

Este estudo justifica-se uma vez que o avanço tecnológico e o surgimento de novas descobertas na área da obstetrícia acabaram por influenciar a maneira como os enfermeiros obstétricos prestam a assistência, e isto acaba gerando conflitos e repercutindo negativamente na atenção.

\section{METODOLOGIA}

Esse artigo é um estudo de revisão integrativa da literatura de natureza descritiva com abordagem qualitativa.

A procura de artigos científicos foi realizada nas bases de dados Scientfic Eletronic Library 
(SciELO) e Biblioteca Virtual da Saúde (BVS). Também foram consultados manuais do MS, portarias e protocolos assistências de diferentes estados como, Rio de Janeiro, Belo Horizonte e Bahia.

Foram selecionados inicialmente vinte artigos publicados entre 2010 e 2018, utilizando os descritores protocolos assistenciais, humanização do parto e boas práticas. Foram excluídos os artigos que não estavam de acordo com os critérios de inclusão ou que fugiam do tema abordado.

Os dados foram coletados no período de janeiro a maio de 2019 e, após a aplicação dos critérios de inclusão e considerando os objetivos do estudo, o número de artigos ficou reduzido a 15.

Após uma leitura exploratória para a procura dos pontos de concordância e discordância entre os autores, foram estabelecidas as seguintes categorias de análise: humanização da assistência ao parto, atuação de enfermeiros obstétricos na atenção ao parto e ao nascimento e protocolos assistenciais ao parto.

\section{RESULTADOS E DISCUSSÃO}

Para a análise dessa revisão foram utilizados 15 artigos, descrevendo aspectos fundamentais para uma assistência segura ao parto e nascimento, incluindo protocolos assistenciais utilizados pelos enfermeiros obstétricos e a humanização ao parto.

Nesta seção descrevemos os resultados obtidos a partir da análise secundária dos artigos selecionados, que estão ilustrados no Quadro 1, organizados por autor, ano de publicação, título e metodologia utilizada nas pesquisas.

Quadro 1. Distribuição dos artigos analisados segundo autor, ano, título, metodologia e base de dados.

\begin{tabular}{|l|l|l|c|c|}
\hline \multicolumn{1}{|c|}{ Autor } & Ano & \multicolumn{1}{|c|}{ Título } & Método & $\begin{array}{c}\text { Base de } \\
\text { Dados }\end{array}$ \\
\hline $\begin{array}{l}\text { BATISTA, B. } \\
\text { D. et al. }\end{array}$ & 2017 & $\begin{array}{l}\text { Fatores associados à satisfação do } \\
\text { acompanhante com o cuidado prestado à } \\
\text { parturiente }\end{array}$ & Estudo transversal & BVS \\
\hline $\begin{array}{l}\text { FERREIRA, A. } \\
\text { G. N. et al. }\end{array}$ & 2013 & $\begin{array}{l}\text { Humanização do parto e nascimento: } \\
\text { acolher a parturiente na perspectiva } \\
\text { dialógica de Paulo Freire }\end{array}$ & Estudo qualitativo & SciELO \\
\hline $\begin{array}{l}\text { GOMES, A. R. } \\
\text { M. et al. }\end{array}$ & 2014 & $\begin{array}{l}\text { Assistência de enfermagem obstétrica na } \\
\text { humanização do parto normal }\end{array}$ & $\begin{array}{l}\text { Revisão } \\
\text { bibliográfica }\end{array}$ & SciELO \\
\hline $\begin{array}{l}\text { GONÇALVES, } \\
\text { R. et al. }\end{array}$ & 2011 & $\begin{array}{l}\text { Vivenciando o cuidado no contexto de } \\
\text { uma casa de parto: o olhar das usuárias }\end{array}$ & $\begin{array}{c}\text { Abordagem } \\
\text { metodológica } \\
\text { qualitativa }\end{array}$ & SciELO \\
\hline $\begin{array}{l}\text { MALHEIROS, } \\
\text { P. A. et al. }\end{array}$ & 2012 & $\begin{array}{l}\text { Parto e Nascimento: saberes e práticas } \\
\text { humanizadas } \\
\text { desritiva, } \\
\text { exploratória }\end{array}$ & SciELO \\
\hline $\begin{array}{l}\text { MELO, B. M. } \\
\text { et al. }\end{array}$ & 2017 & $\begin{array}{l}\text { Implementação das boas práticas na } \\
\text { atenção ao parto em maternidade de } \\
\text { referência }\end{array}$ & $\begin{array}{l}\text { Abordagem } \\
\text { quantitativa }\end{array}$ & BVS \\
\hline $\begin{array}{l}\text { MELO, G. P. } \\
\text { et al. }\end{array}$ & 2016 & $\begin{array}{l}\text { Elaboração e validação do protocolo } \\
\text { assistencial de enfermagem para sala de } \\
\text { préparto, parto e pós-parto* }\end{array}$ & $\begin{array}{l}\text { Pesquisa } \\
\text { metodológica }\end{array}$ & SciELO \\
\hline $\begin{array}{l}\text { NICOLINI, A. } \\
\text { B. et al. }\end{array}$ & 2017 & $\begin{array}{l}\text { Processo de elaboração de protocolo para } \\
\text { assistência humanizada de enfermagem } \\
\text { ao parto de risco habitual }\end{array}$ & $\begin{array}{l}\text { Pesquisa } \\
\text { qualitativa }\end{array}$ & SciELO \\
\hline $\begin{array}{l}\text { POSSATI, A. } \\
\text { B. et al. }\end{array}$ & 2017 & $\begin{array}{l}\text { Humanização do parto: significados e } \\
\text { percepções de enfermeiras }\end{array}$ & $\begin{array}{l}\text { Pesquisa } \\
\text { qualitativa } \\
\text { descritiva }\end{array}$ & $\begin{array}{l}\text { Pesquisa } \\
\text { qualitativa }\end{array}$ \\
\hline $\begin{array}{l}\text { RAMOS, W. } \\
\text { M. A. et al. }\end{array}$ & 2018 & $\begin{array}{l}\text { Contribuição da enfermeira obstétrica nas } \\
\text { boas práticas da assistência ao parto e } \\
\text { nascimento }\end{array}$ & BVS \\
\hline
\end{tabular}




\begin{tabular}{|l|l|l|c|c|}
\hline $\begin{array}{l}\text { RABELO, L. } \\
\text { R.; OLIVEIRA, } \\
\text { D. L. }\end{array}$ & 2010 & $\begin{array}{l}\text { Percepções de enfermeiras obstétricas } \\
\text { sobre sua competência na atenção ao } \\
\text { parto normal hospitalar }\end{array}$ & $\begin{array}{l}\text { Pesquisa } \\
\text { qualitativa }\end{array}$ & SciELO \\
\hline $\begin{array}{l}\text { REIS, T. R. et } \\
\text { al. }\end{array}$ & 2015 & $\begin{array}{l}\text { Enfermagem obstétrica: contribuições às } \\
\text { metas dos Objetivos de Desenvolvimento } \\
\text { do Milênio }\end{array}$ & Estudo quantitativo & SciELO \\
\hline $\begin{array}{l}\text { SANTOS, R. } \\
\begin{array}{l}\text { A. A.; MELO, } \\
\text { M. C. P.; } \\
\text { CRUZ, D. D. }\end{array}\end{array}$ & 2015 & $\begin{array}{l}\text { Trajetória de humanização do parto no } \\
\text { Brasil a partir de uma revisão integrativa } \\
\text { de literatura }\end{array}$ & $\begin{array}{l}\text { Revisão } \\
\text { de literatura } \\
\text { integrativa }\end{array}$ & SciELO \\
\hline $\begin{array}{l}\text { SILVA, T. C. et } \\
\text { al. }\end{array}$ & 2017 & $\begin{array}{l}\text { Práticas de atenção ao parto e } \\
\text { nascimento: uma revisão integrativa }\end{array}$ & Revisão integrativa & BVS \\
\hline $\begin{array}{l}\text { VIEIRA, M. } \\
\text { J.O. et al. }\end{array}$ & 2016 & $\begin{array}{l}\text { Assistência de enfermagem obstétrica } \\
\text { baseada em boas práticas: do acolhimento } \\
\text { ao parto }\end{array}$ & $\begin{array}{l}\text { Descritivo, } \\
\text { retrospectivo e } \\
\text { documental, } \\
\text { abordagem } \\
\text { quantitativa }\end{array}$ & SciELO \\
\hline
\end{tabular}

Fonte: Dados da pesquisa, 2018.

Conforme o Quadro 1, o maior número de artigos (5) foi publicado em 2017, seguido do anos 2015 e 2016, ambos com 02 (dois) artigos cada. Quanto ao tipo de pesquisa, 10 foram qualitativos e 05 quantitativos, mostrando uma preponderância de pesquisas qualitativas na área. Já com relação às bases de dados, foram encontrados 11 artigos na base SciELO e 4 artigos na BVS.

Os periódicos nos quais foram publicados os artigos selecionados estão listados no Quadro 2 conforme a quantidade e percentual. As publicações estão equilibradamente distribuidas em revistas da área de Enfermagem. Apenas a Revista da Escola de Enfermagem da USP e a Revista Eletrônica de Enfermagem tiveram um número maior de artigos (2), o que corresponden a 13,3\%.

Quadro 2. Periódicos analisados conforme a quantidade e percentagem de artigos publicados.

\begin{tabular}{|l|c|c|}
\hline \multirow{2}{*}{ Períodicos } & \multicolumn{2}{c|}{ Artigos } \\
\cline { 2 - 3 } & Quantidade & Porcentagem (\%) \\
\hline Cogitare Enfermagem & 1 & $6,7 \%$ \\
\hline Revista de Enfermagem UFPE & 1 & $6,7 \%$ \\
\hline Revista Científica de Enfermagem-Recien & 1 & $6,7 \%$ \\
\hline Revista da Escola de Enfermagem da USP & 2 & $13,3 \%$ \\
\hline Texto Contexto Enfermagem & 1 & $6,7 \%$ \\
\hline Revista da Rede de Enfermagem do Nordeste-Rene & 1 & $6,7 \%$ \\
\hline Escola Anna Nery & 1 & $6,7 \%$ \\
\hline Revista Online de Pesquisa Cuidado é Fundamental & 1 & $6,7 \%$ \\
\hline Revista Gaúcha de Enfermagem - RGE & 1 & $6,7 \%$ \\
\hline Caderno de Cultura e Ciência & 1 & $6,7 \%$ \\
\hline Revista de Enfermagem do Centro-Oeste Mineiro & 1 & $6,7 \%$ \\
\hline Revista Eletrônica de Enfermagem & 2 & $13,3 \%$ \\
\hline Revista Ciência, Cuidado e Saúde & 1 & $6,7 \%$ \\
\hline
\end{tabular}

Fonte: Dados de pesquisa, 2018.

Após a análise dos artigos, foi possível organizar as informações mais relevantes sobre os protocolos assistenciais utilizados pelos enfermeiros obstétricos na promoção da segurança e da humanização no cuidado à parturiente e organizá-las de acordo com as categorias mencionadas anteriormente e apresentadas a seguir. 


\section{Humanização da assistência ao parto}

Segundo Gomes et al. (2014), o excessivo uso de medicamentos e tecnologias na assistência ao parto no decorrer da história tornou este processo, antes natural, em um parto medicalizado e intervencionista, contribuindo para o aumento dos índices de morbimortalidade materna e perinatal.

Estes altos índices, decorrentes de práticas intervencionistas, acarretaram um sério problema de saúde pública, devido ao grande número de intervenções desnecessárias, levando a complicações que, muitas vezes, podem ser irreversíveis ou evoluir em óbito materno e/ou perinatal. Com isso, as ações humanizdas foram sendo deixadas de lado, tendo como consequência o surgimento das violências obstétricas $^{6}$.

Para Gomes et al. (2014) e Silva et al. (2017), a humanização da assistência ao parto implica respeito aos direitos das mulheres e crianças, que inclui o bem estar físico e psicológico, a segurança e o conforto. Deve-se incluir também uma boa relação interpessoal entre profissionais, pacientes e acompanhantes. Possati et al. (2017) acrescentam que, para que haja uma ação de fato humanizada, é necessário saber acolher, ouvir a parturiente e sua família, orientá-los e criar vínculos interpessoais.

Diante do que vem sendo apresentado sobre acolhimento humanizado a gestantes e familiares, fica nítido que, para uma assistência com essas características, deve-se ter como princípios fundamentais o conhecimento, a prática e condutas baseadas em evidências, que venham a contribuir para um parto e nascimento saudáveis, que reduçam os indicadores de morbimortalidade materna e perinatal (BRASIL, 2001; FERREIRA, 2013; POSSATI, 2017; SILVA, 2017).

Estudos como Brasil (2001), Santos, Melo e Cruz (2015) e Silva et al. (2017) relatam ainda que para uma assistência de qualidade e humanizada no parto e nascimento é importante que tais ações sejam iniciadas ainda no pré-natal. Logo, os profissionais de saúde devem orientar, informar e tirar as possíveis dúvidas das mulheres no ciclo gravídico-puerperal, evitando futuras intervenções desnecessárias e garantindo que estas mulheres tenham autonomia durante todo o processo.

Em consonância com os pontos mencionados, Gomes et al. (2014) apresentam algumas diretrizes formuladas pelo MS e pela OMS que devem ser adotadas no pré-natal, para que haja uma melhor assistência durante o parto. Elas consistem em planejamento do nascimento e parto, avaliação de riscos e intercorrências que podem surgir, respeito às escolhas da parturiente, bem como a imperatividade de fornecimento de informações às mulheres e familiares.

Diante dos aspectos negativos do processo da medicalização do parto, foram criadas pelo MS algumas políticas estimuladoras para um parto natural e humanizado como, por exemplo, a Rede Cegonha criada em 2011 e o PHPN criado em 2000, para que o parto normal seja uma escolha esclarecida e segura para a mulher (SANTOS; MELO; CRUZ, 2015).

Silva et al. (2017) explicam que o PHPN foi criado com o intuito de melhorar a assistência obstétrica, tornando-a mais qualificada, humanizada e integral, desde o pré-natal e parto até o puérperio. Possati et al. (2017) ainda acrescentam que o PHPN trouxe novas práticas, todas baseadas em evidências científicas, como o direito a acompanhante, qualificação dos profissionais, bem como uma melhor relação interpessoal entres profissionais e parturientes, de forma a promover a autonomia e protagonismo da mulher de acordo com seus direitos.

Outra medida do MS foi a Lei 11.108 de 2005, que proporciona à mulher o direito a ter um acompanhante de sua escolha durante o trabalho de parto, parto e puerpério (SANTOS; MELO; CRUZ, 2015). Ressalta-se que o acompanhante tem uma função importante neste período, já que, como foi comprovado, ter uma pessoa de confiança ao lado da mulher na hora do parto pode gerar inúmeros benefícios, pois traz apoio emocional, conforto, segurança e tranquilidade à parturiente (GOMES, 2014; MELO, 2017; SILVA, 2017).

Batista et al. (2017) afirmam que a presença do acompanhante contribui com o empoderamento da

${ }^{6}$ Violências obstétricas são práticas desumanizadas que acontecem na gestação, parto, nascimento e pós-parto, em que as mulheres são submetidas a rotinas desnecessárias, que desrespeitam o seu corpo a as impedem de exercer seu protagonismo. 
mulher durante seu parto e permite que esta fique mais calma e forte, resultando em uma diminuição do tempo de trabalho de parto e redução da dor. Consequentemente, a mulher fica mais satisfeita com a experiência vivida, acarreta melhora no $\operatorname{Apgar}^{7}$ do neonato e diminui as chances de intervenções desnecessárias e violências obstétricas.

Baseando-se no que foi abordado nesta categoria, pode-se concluir que o processo de humanização requer a qualificação do enfermeiro obstétrico para assistir a parturiente de forma satisfatória em todo o processo do trabalho de parto, parto e pós-parto, utilizando os protocolos assistenciais baseados em evidências científicas.

\section{Atuação de enfermeiros obstétricos na atenção ao parto e ao nascimento}

O parto é um evento natural que, com a evolução científica, sofreu a interferência de novas técnicas e procedimentos que modificaram o processo de forma nem sempre benefica para a parturiente e seu feto. Contudo, também houve evolução das políticas públicas de saúde da mulher, que tentam resgatar a essência do parto natural, a atenção holística e humanizada e, principalmente, oportunizaram a conscientização dos profissionais da obstetrícia no que diz respeito a prestar uma atenção ao parto e nascimento por meio de práticas simples, baseadas em evidências científicas, incentivando o parto normal e minimizand o o índice de morbimortalidade materna e perinatal.

Os profissionais de saúde possuem um papel muito importante durante o parto. É nesse momento que todo o conhecimento deve ser utilizado para assegurar o bem estar da mulher e do neonato, pois eles devem saber reconhecer o momento certo de intervir em casos de necessidade, promovendo, assim, o conforto e saúde dos mesmos. Além disso, estes devem estar prontos para ofertar apoio, orientação, força e todo suporte necessário nesse momento que é único na vida de cada mulher (BRASIL, 2001).

Para Gomes et al. (2014), os enfermeiros obstétricos são reconhecidos como pessoas capazes de acompanhar mulheres no ciclo gravídico-puerperal de baixo risco, devido a sua capacidade de prestar uma assistência de qualidade, segura e humanizada. Ramos et al. (2018) ainda acrescentam que estes profissionais possuem um diferencial na sua assistência por possuírem características próprias como o respeito, conhecimentos e a constante busca de evidências científicas, o que torna a profissão cada vez mais forte e reconhecida e a assistência mais segura, qualificada e humanizada.

Os enfermeiros obstétricos devem encorajar as mulheres a serem protagonistas, incentivando a participação ativa da parturiente no parto. Devem também estimular procedimentos como o contato pele a pele entre o binômio mãe-filho, o clampeamento oportuno do cordão umbilical com a participação do acompanhante, a amamentação logo após o nascimento e, principalmente, respeito à hora dourada que é a primeira hora após o nascimento (RAMOS, 2018).

Ao longo dos anos, estes profissionais vêm sendo qualificados através de especializações e respaldados por resoluções, a exemplo a Resolução do COFEN n 0516/2016, que assegura e permite que os partos normais sejam assistidos por enfermeiros obstétricos e obstetrizes. Esta resolução incentiva, ainda, a criação de casas ou centros de parto normal, tendo como atuantes os enfermeiros obstétricos (GOMES, 2014).

Para Gomes et al. (2014), questões como estimular à parturiente na deambulação, evitar o uso rotineiro da ocitocina e episiotomia e o encorajamento ao parto vertical são procedimentos que ainda causam discordância entre os profissionais. Por esse motivo, é de suma importância que sejam ofertadas capacitações para os enfermeiros obstétricos que ajudem a superar as divergências, e promovam o respeito aos direitos das mulheres.

Visto que há evidências científicas dos benefícios que as práticas humanizadas trazem para o parto e nascimento, os enfermeiros obstétricos precisam estar atualizados e em constante aprimoramento,

${ }^{7}$ O Apgar é um teste realizado em recém-nascidos, cujo objetivo é avaliar a vitalidade do neonato ao nascer e detectar possíveis complicações. Esse teste é realizado no primeiro e quinto minutos de vida, possui cinco sinais de avaliação (freqüência cardíaca, respiração, tônus muscular, cor e irritabilidade reflexa), sendo que a cada um destes sinais é atribuído uma pontuação de 0 a 2 pontos. Quanto mais alta a pontuação, menores são os riscos para o neonato. 
para que haja uma melhora no modelo de cuidado à saúde da mulher (RAMOS, 2018), já que no modelo de atenção ao parto e nascimento em nosso país ainda predominam as ações intervencionistas que dificultam o empoderamento da mulher em seu parto (VIEIRA, 2016).

As boas práticas no parto e nascimento compreendem questões como o cuidado físico, a realização de procedimentos que tragam benefícios, tanto à parturiente como ao feto, redução de procedimentos desnecessários, garantia da privacidade, da autonomia e dos direitos da mulher, bem como aspectos abordados pelo PHPN (FERREIRA, 2013). Estas são algumas práticas que, quando executadas pelos enfermeiros obstétricos, indicam que estes estão prestando uma assistência qualificada e humanizada, respeitando as necessidades individuais de cada mulher e reconhecendo seu protagonismo durante este processo, que vai desde o pré-natal até o puerpério e, principalmente, seguindo as práticas baseadas em evidências científicas, preconizadas pelo MS e OMS, que servem de embasamento para a formulação dos protocolos assistenciais das instituições.

Ferreira et al. (2013) afirmam que as divergências causadas pelas diferentes crenças e valores dos involucrados na gestação e parto geram receio por parte da parturiente na hora de tirar suas dúvidas e comunicar-se, dando origem a sua submissão no parto. Diante disto, Silva et al. (2017) explicam que o modelo de cuidado utilizado pelos enfermeiros obstétricos está embasado nas condutas de humanização da assistência à mulher e ao recém-nascido, respaldado pelas políticas públicas de saúde, que trazem práticas apropriadas, pertinentes e com fundamentação teórica, de modo a valorizar a cultura, crenças e singularidades de cada mulher.

O empoderamento e autonomia conquistados pelas mulheres nas últimas décadas contribuiram positivamente para o surgimento de estratégias voltadas à saúde da mulher. As ações de educação em saúde, junto à contribuição dos profissionais, em especial dos enfermeiros obstétricos, possibilitaram o acesso das mulheres no ciclo gravídico-puerperal a uma gama de informações e serviços que lhes proporcionam um melhor entendimento acerca de questões como a gravidez, o parto normal, planejamento familiar, saúde sexual e reprodutiva, entre outras demandas. E essa compreensão é fundamental para que a mulher possa assumir o seu papel protagônico durante a gestação e parto.

\section{Protocolos assistenciais ao parto}

Os protocolos assistenciais contribuem com a melhoria da assistência, tornando-a mais qualificada, padronizada e organizada, além de atualizar os profissionais quanto às novas práticas de assistência ao pré-parto, parto e puerpério. Estes protocolos são elaborados a partir de evidências científicas que garantem uma assistência com condutas seguras e atuais, sem intervenções desnecessárias e com resultados satisfatórios para todos (MELO, 2016; NICOLINI, 2017).

No que diz respeito à atuação do enfermeiro obstétrico, o parágrafo único da Lei nº 7.498/86 afirma que aos enfermeiros obstétricos incumbe a "assistência à parturiente e ao parto normal, identificação das distócias obstétricas e tomada de providências até a chegada do médico, realização de episiotomia e episiorrafia e aplicação de anestesia local, quando necessária" (COREN, 2013, p.49).

A Resolução COFEN n 0516/2016, que dispõe sobre a atuação e responsabilidade dos enfermeiros, enfermeiros obstétricos e obstetrizes na assistência a gestantes, parturientes, puérperas e recém-nascidos, estabelece em seu artigo $3^{\circ}$ e parágrafo único as competências destes profissionais, dentre elas o acolhimento à mulher, acompanhantes e familiares, realização de práticas baseadas em evidências científicas, avaliação de condições clínicas e obstétricas materna e fetal, avaliação da evolução do trabalho de parto, assistência ao parto normal (fisiológico), registro de todas as informações em prontuário, notificação de óbitos maternos e neonatais, identificação de distócias e tomada de providências até a chegada do médico, realização da episiotomia, episiorrafia e aplicar de anestesia local, quando necessário e assistência obstétrica a mulher e neonato desde a internação até a alta (COFEN, 2016).

Para facilitar o processo de conhecimento das atribuições da enfermagem obstétrica, realizou-se um detalhamento suscinto e correlacionado de alguns protocolos de assistência ao parto, utilizados nos 
estados de Bahia, Rio de Janeiro e Minas Gerais, a partir de dados da Associação Brasileira de Obstetrizes e Enfermeiros Obstetras (ABENFO) de 2013, 2014 e 2015.

O protocolo de assistência do estado da Bahia tem como objetivo apresentar diretrizes que possam nortear os profissionais a prestarem uma assistência integral e individualizada a cada mulher, incentivando o exercício das boas práticas no ciclo grávido-puerperal, bem como contribuir para o fortalecimento da enfermagem obstétrica (ABENFO, 2014). Já o protocolo do Rio de Janeiro objetivou definir diretrizes norteadoras da prática de enfermagem obstétrica, assim como configurar uma relação entre profissionais de diferentes categorias com o intuito de ter uma prática assistencial adequada para cada mulher (ABENFO, 2013). Quanto a Minas Gerais, o protocolo tem como objetivo evidenciar a importância do protagonismo da mulher no seu parto, considerado como um processo fisiológico, prestar uma assistência multiprofissional com foco no bem estar e segurança materna e neonatal, além de assegurar uma assistência baseada em evidências científicas, livre de práticas desnecessárias (ABENFO, 2015).

Todos os três protocolos foram criados para serem usados em conjunto com outras publicações, como portarias e resoluções do MS, do Conselho Federal de Enfermagem (COFEN), Conselho Regional de Enfermagem (COREN), ABENFO, entre outros.

Os objetivos dos protocolos assistenciais aqui analisados assemelham-se e complementam-se na direção de conduzir o trabalho da enfermagem obstétrica de forma eficaz e multidisciplinar, reconhecendo as suas potencialidades e competências acadêmicas e científicas.

Quanto ao processo de atendimento e assistência à gestante, os três protocolos apresentaram medidas semelhantes e de relevância. No que se refere à assistência durante a admissão, é preciso avaliar alguns aspectos de cada mulher, como físico, obstétrico, familiar e psicológico, além do histórico da gestante, para que possa haver um parto e nascimento fisiológicos (ABENFO, 2014).

A parturiente, ao chegar à admissão, precisa ser acolhida e passar pela classificação de risco, suas queixas devem ser ouvidas, seus sinais vitais registrados e tanto a mulher quanto o acompanhante devem responder algumas perguntas a respeito do histórico da mulher e de sua gravidez. Deverá também ser realizada a leitura do cartão de pré-natal, atentando para os exames laboratoriais e complementares apresentados relacionando-os com a clínica obstétrica. É importante investigar os achados patológicos e, finalmente, de acordo com os critérios de risco habitual, a gestante é admitida (ABENFO, 2014). Este primeiro contato é muito importante para detecção de possíveis anormalidades.

ABENFO (2015) acrescenta que o acolhimento nesse primeiro contato com a maternidade é muito importante. É na admissão que é criado um vínculo de confiança entre a mulher e os profissionais. É neste momento que são reconhecidos detalhes importantes que irão contribuir para o protagonismo daquela mulher e para um bom parto e nascimento.

Ainda de acordo com a ABENFO $(2013,2014)$, após a admissão, a parturiente deve passar por exame físico com inspeção da pele e mucosa, tórax, região abdominal, aparelho geniturinário e membros inferiores. Devem ser realizada as Manobras de Leopold ${ }^{8}$ e ausculta da frequência cardíaca fetal, avaliação do padrão da contratilidade uterina, avaliação da vulva e toque vaginal, aproveitando para verificar a integridade das membranas, avaliação da dilatação, apagamento e posição do colo, além de avaliar a apresentação fetal, altura e variedade de posição.

Corroborando, a ABENFO (2015) explica que os profissionais devem ainda prestar informações à mulher e seu acompanhante a respeito do diagnóstico do trabalho de parto e rotinas da instituição, bem como prestar apoio a ambos durante todo o processo, atentar para o plano de parto apresentado pela mulher, respeitando suas escolhas, realizar teste para sífilis e Vírus da Imunodeficiência Humana (HIV), realizar registro no partograma, incentivar a presença do acompanhante, a posição para trabalho de parto e parto, a deambulação, as posições verticalizadas, a dieta a base de líquido e, principalmente, evitar manobras e intervenções desnecessárias.

${ }^{8}$ Manobras de Leopold são técnicas utilizadas para identificar o crescimento fetal, determinar sua situação (longitudinal, transversa ou oblíqua), sua apresentação (cefálica, pélvica ou côrnica) e posição (direita ou esquerda). 
Os protocolos divergem um pouco a respeito da dilatação cervical no início do trabalho de parto ativo: o da Bahia afirma ser dilatação de $4 \mathrm{~cm}$ com colo completamente apagado ou $5 \mathrm{~cm}$ independente do apagamento; o do Rio de Janeiro afirma que a dilatação é a partir dos $4 \mathrm{~cm}$; e o protocolo de Minas Gerais considera que a dilatação ocorre a partir de 3 cm (ABENFO, 2013, 2014 e 2015).

No período de dilatação devem ser oferecidos a mulher métodos não invasivos para o alívio da dor. As informações devem ser passadas em linguagem clara e aos poucos, para um melhor entendimento e os sinais vitais da parturiente e do feto devem ser verificados, atentando para anormalidades (ABENFO, 2013 e 2014). Ademais, é importante observar o comportamento da mulher, que dá sinais claros desta fase, quando deve-se monitorar aspectos como as contrações uterinas, progresso da dilatação e da descida do bebê, e a ausculta intermitente de batimentos cardíacos fetais (ABENFO, 2013). Práticas como o jejum prolongado, tricotomia, hidratação venosa e amniotomia devem ser evitadas (ABENFO, 2013, 2014 e 2015).,

Melo et al. (2017) apresentam algumas técnicas não farmacológicas para o alívio da dor, como as massagens corporais, os banhos de chuveiro ou imersão, as técnicas de respiração, a deambulação ativa, o uso de bolas e cavalinhos e suporte emocional, ações que contribuem com a redução do uso rotineiro da ocitocina. Acrescentam, ainda, que toda a evolução do trabalho de parto deve ser monitorada pelo partograma, o que permite que qualquer anormalidade seja detectada precocemente e que intervenções sejam adotadas com a finalidade de resolver a situação.

O período de expulsão é quando o enfermeiro obstétrico deve orientar as parturientes quanto à melhor posição para parto, desde que seja confortável e com uma postura verticalizada, que é o mais recomendado. Todos os autores afirmam que os batimentos cardíacos fetais devem ser auscultados a cada 5 minutos, manobras como a de Kristeller ${ }^{9}$ são contra-indicadas. O uso da ocitocina deve ocorrer apenas quando este período não estiver progredindo; o ambiente deve ser preparado para receber o recém-nascido com uma diminuição da intensidade da luz e do tom de voz dos profissionais, que deve ser mais brando; a episiotomia deve ser restrita (ABENFO, 2013, 2014 e 2015).

Além disto, novas práticas baseadas em evidências científicas devem ser adotadas por estes profissionais, como o contato pele a pele entre mãe e bebê, o que fortalece o vínculo afetivo, o clampeamento tardio do cordão umbilical e o incentivo à amamentação na primeira hora após o nascimento (ABENFO, 2013, 2014 e 2015).

Já no período de dequitação ${ }^{10}$, é preciso uma observação adicional dos profissionais por ser um momento no qual pode ocorrer hemorragia. A atenção destes profissionais deve estar voltada para possíveis efeitos adversos, buscando reduzi-los ao máximo, evitando a intervenção no processo fisiológico e a interferência entre o binômio mãe-bebê (ABENFO, 2014).

Além do que, durante este período, o enfermeiro obstétrico pode optar por duas formas de realizar a dequitação. A primeira delas é por conduta expectante, respeitando o processo fisiológico para que a dequitação ocorra naturalmente e realizando o clampeamento tardio do cordão umbilical, optando por intervir se houver complicações. A segunda forma é por conduta ativa para evitar complicações hemorrágicas, fazendo uso de ocitocina, clampeamento, secção precoce e tração controlada do cordão umbilical para desprendimento da placenta (ABENFO, 2014 e 2015).

Conforme evidência científica, é necessária a administração de 10 unidades de ocitocina via intramuscular, independente da conduta adotada, como prevenção para hemorragia (ABENFO, 2013, 2014 e 2015). E após a dequitação, deve-se realizar exame minucioso da placenta, cordão e membranas. Posteriormente, o profissional deve registrar os dados observados em prontuário e, se for da vontade da puérpera, é seu direito poder levar a sua placenta para casa (ABENFO, 2015).

De acordo com ABENFO (2014 e 2015), no período de greenberg ${ }^{11}$, que vai do fim da dequitação até as primeiras horas após o parto, o profissional deve realizar o toque bidigital para verificar se permaneceu algum fragmento de placenta no canal do parto. É um período em que se deve manter a

\footnotetext{
${ }^{9}$ Manobra de Kristeller é uma técnica utilizada durante o parto, na qual é aplicada uma pressão com os braços, na parte superior do útero, com objetivo de facilitar a saída do feto.

${ }^{10}$ Dequitação é o período de separação e expulsão da placenta.

${ }^{11}$ Greenberg é o período que começa após a dequitação e vai até a primeira ou segunda hora pós-parto.
} 
atenção ainda na puérpera, por conta dos riscos de hemorragia e também deve ser um momento de fortalecimento de vínculo entre mãe e bebê, sendo contraindicado a separação de ambos.

ABENFO (2014) elenca alguns cuidados básicos que devem ser executados pelos enfermeiros obstétricos:

\begin{abstract}
Identificar alteração do padrão de sangramento e intervir no sentido de controle, com massagens e uso de medicação se necessário (ocitocina ou ergometrina); monitorar sinais vitais de 30 em 30 minutos; estimular, apoiar, orientar e ajudar no aleitamento materno; observar involução uterina e a formação do globo de Pinnard; observar mucosas; reconhecer precocemente palidez, taquicardia ou hipotensão, que podem indicar hemorragia; em caso de alguma alteração comunicar imediatamente médico obstetra (ABENFO, 2014, p.59).
\end{abstract}

Desta maneira, destaca-se que as primeiras horas de vida para o recém-nascido são importantes e devem ser respeitadas. Neste período, também chamado de período sensível, o bebê está alerta e faz contato visual, o contato pele a pele com sua genitora, o cheiro dela e a amamentação consolidam os laços afetivos (ABENFO, 2013 e 2015).

ABENFO (2014) também elenca ações relacionados à atenção ao recém-nascido:

\begin{abstract}
Verificar a existência do cartão da criança e seu adequado preenchimento; verificar os dados do RN ao nascer (peso, estatura, APGAR, condições de vitalidade); observar e orientar a genitora quanto a mamada, reforçando as orientações dadas durante o prénatal, destacando a necessidade do aleitamento materno exclusivo até o sexto mês de vida do bebê; observar e avaliar a mamada para garantia de adequado posicionamento e pega correta; observar a criança em relação ao peso, postura, atividade espontânea, padrão respiratório, estado de hidratação, eliminações, aleitamento materno, características da pele (presença de palidez, icterícia e cianose), crânio, orelhas, olhos, nariz, boca, pescoço, tórax, abdômen (condições de coto umbilical), genitália, extremidades e coluna vertebral. Caso seja detectada alguma alteração, solicitar avaliação médica imediatamente; orientar a genitora quanto ao teste do pezinho, olhinho, orelhinha e consulta de puericultura; encaminhar para tomar vacinas (BCG e contra hepatite B) (ABENFO, 2014, p 65).
\end{abstract}

Os cuidados no puerpério incluem a verificação de queixas da mulher, inspeção da dor, checar sinais vitais (pulso, temperatura, pressão arterial, freqüência respiratória), inspecionar também condição das mucosas e pele, eliminações, características e presença de fluxo vaginal logo após o parto (lóquios) como cor, cheiro e quantidade, genitais e períneo (verificar a higiene, cicatrização de episiorrafia se houver, sinais de infecção, presença de edema e hematomas), o estado das mamas (verificar a presença de ingurgitamento mamário, sinais inflamatórios, infecciosos ou cicatrizes que dificultem a amamentação, conformação dos mamilos, presença de fissuras). O estado emocional da puérpera deve ser avaliado, observando se há aceitação da maternidade, se há um bom vínculo mãe-bebê, se há boa integração entre mãe-pai-bebê e demais membros da família, sempre à procura de sinais que possam indicar o estado emocional, para avaliar o risco de depressão pós-parto (ABENFO, 2014 e 2015).

\title{
CONSIDERAÇÕES FINAIS
}

Os enfermeiros obstétricos possuem total liberdade para assistir no parto, identificar complicações, realizar encaminhamentos e procedimentos pertinentes durante o ciclo gravídicopuerperal, inclusive embasados pela legislação e nos protocolos assistenciais já existentes. Contudo, a utilização de protocolos assistências tem como finalidade auxiliar e orientar os enfermeiros obstétricos 
quanto à sua atuação no pré-parto, parto e puerpério. Eles são de suma importância para uma assistência segura, qualificada e humanizada, garantindo aos profissionais maior autonomia e reconhecimento de suas competências, além de ser um guia de conhecimento.

Assim, fica evidente que a assistência obstétrica dirigida por protocolos assistenciais, atrelado às práticas humanizadas, trazem uma redução significativa no índice de morbimortalidade materna e perinatal, uma vez que os partos normais ou naturais estimulam um processo que é fisiológico da mulher e elas devem ser estimuladas a serem protagonistas deste processo. Além disto, existem técnicas que auxiliam no trabalho de parto, proporcionando à mulher a redução da dor. Também, é bastante estimulador que lhe seja concedido o direito de ter um acompanhante e de escolher a melhor posição para o parto. Essas técnicas têm garantido o bem estar da parturiente e um parto de qualidade, humanizado e seguro.

Portanto, ao relacionar os protocolos assistenciais utilizados pelos enfermeiros obstétricos que promovem a segurança e humanização do parto, evidenciou-se que os protocolos estão seguindo uma mesma linha em prol do que é melhor para a parturiente e o feto ao evitar procedimentos desnecessários. Logo, aspira-se a que, gradativamente, as boas práticas se propaguem e que os profissionais obstétricos se conscientizem e se aperfeiçoem para possibilitar às mulheres que procuram o serviço público de saúde uma assistência mais adequada e segura.

\section{REFERÊNCIAS}

ABENFO. Assistência ao parto e nascimento diretrizes para o cuidado multidisciplinar. Belo Horizonte, 2015. Disponível em <https://www.ABENFO.org.br/site/biblioteca/arquivos/manuais/241_ProtocoloAssistencia_Parto_Nascimento-18-12-2015.pdf>. Acesso dia 13 Mar. 2018.

ABENFO. Protocolo Assistencial da Enfermagem Obstétrica da Secretaria Municipal SMS/RJ. Rio de janeiro, 2013. Disponível em <http://redesindical.com.br/ABENFO/arqs/manuais/161.pdf>. Acesso dia 13 Mar. 2018.

ABENFO. Protocolo Assistencial da Enfermeira Obstetra no Estado da Bahia. Salvador-BA, 2014. Disponivel em: <http://ba.corens.portalcofen.gov.br/wp-content/uploads/2014/12/Protocolo_Enfermagem.pdf> . Acesso dia 12 Mar. 2018.

BATISTA, B. D. et al. Fatores associados à satisfação do acompanhante com o cuidado prestado à parturiente. Cogitare Enferm. (22)3: e51355, 2017. Disponível em: <http://pesquisa.bvsalud.org/portal/resource/pt/biblio875383>. Acesso dia 25 Mar. 2018.

BRASIL. Ministério da Saúde. Portaria nº 1.459 de 24 de junho de 2011. Institui no âmbito do Sistema Único de Saúde - SUS - a Rede Cegonha. Disponível em <http://bvsms.saude.gov.br/bvs/saudelegis/gm/2011/prt1459_24_06_2011.html>. Acesso em: 23 Jan. 2018.

BRASIL. Ministério da Saúde. Secretaria de Políticas de Saúde. Área Técnica de Saúde da Mulher. Parto, aborto e puerpério: assistência humanizada à mulher. Brasília-DF, 2001. Disponível em: <http://bvsms.saude.gov.br/bvs/publicacoes/cd04_13.pdf>. Acesso em: 22 Jan. 2018.

BRASIL. Ministério da Saúde. Secretaria de Ciência, Tecnologia e Insumos Estratégicos. Departamento de Gestão e Incorporação de Tecnologias em Saúde. Diretrizes Nacionais de Assistência ao Parto Normal: versão resumida [recurso eletrônico] - Brasília-DF, 2017. Disponível em <http://bvsms.saude.gov.br/bvs/publicacoes/diretrizes_nacionais_assistencia_parto_normal.pdf $>$. Acesso em: 22 Jan. 2018.

COREN. Legislação Básica para o exercício de Enfermagem. Conselho Regional de Enfermagem (COREN)BA. $4^{a}$ Ed. 2013. 48-49p. Disponível em: <http://ba.corens.portalcofen.gov.br/wpcontent/uploads/2013/07/CARTILHA_LEGISLACAO_COREN.pdf>. Acesso em: 09 Mar. 2018.

COFEN. Conselho Federal de Enfermagem (COFEN). Resolução $n^{\circ}$ 0516/2016. Disponível em: <http://www.cofen.gov.br/resolucao-cofen-no-05162016_41989.html>. Acesso em: 09 Mar. 2018. 
FERREIRA, A. G. N. et al. Humanização do parto e nascimento: acolher a parturiente na perspectiva dialógica de Paulo Freire. Rev enferm UFPE on line., Recife, 7(5):1398-405, maio., 2013. Disponível em:

$<$ http://repositorio.ufc.br/ri/handle/riufc/5642>. Acesso em: 07 Mar. 2018.

GOMES, A. R. M. et al. Assistência de enfermagem obstétrica na humanização do parto normal. São Paulo:

Revista Recien. 2014; 4(11):23-27. Disponível em:

<http://www.recien.com.br/index.php/Recien/article/view/73/137>. Acesso dia 23 Jan. 2018.

GONÇALVES, R. et al. Vivenciando o cuidado no contexto de uma casa de parto: o olhar das usuárias. Rev Esc Enferm USP 2011; 45(1):62-70 www.ee.usp.br/reeusp/. Disponível em:

<http://www.scielo.br/pdf/reeusp/v45n1/09.pdf>. Acesso em: 22 Jan. 2018.

MALHEIROS, P. A. et al. Parto e Nascimento: saberes e práticas humanizadas. Texto Contexto Enferm, Florianópolis, 2012 Abr-Jun; 21(2): 329-37. Disponível em: <http://www.scielo.br/pdf/tce/v21n2/a10v21n2>. Acesso dia 22 Jan. de 2018.

MELO, B. M. et al. Implementação das boas práticas na atenção ao parto em maternidade de referência. Rev Rene. 2017 maio-jun; 18(3):376-82. Disponível em:_<http://pesquisa.bvsalud.org/portal/resource/pt/biblio849265>. Acesso dia 08 Mar. 2018.

MELO, G. P. et al. Elaboração e validação do protocolo assistencial de enfermagem para sala de pré- parto, parto e pós-parto*. Rev. Eletr. Enf. [Internet]. 2016. Disponível em:

<https://revistas.ufg.br/fen/article/view/40589>. Acesso dia 17 Mai. 2018.

NICOLINI, A. B. et al. Processo de elaboração de protocolo para assistência humanizada de enfermagem ao parto de risco habitual. Cienc Cuid Saude 2017 Out-Dez; 16(4). Disponível em:

<http://periodicos.uem.br/ojs/index.php/CiencCuidSaude/article/view/36841>. Acesso dia 17 Mai. 2018.

POSSATI, A. B. et al. Humanização do parto: significados e percepções de enfermeiras. Esc Anna Nery

2017;21(4):1-6. Disponível em: <http://www.redalyc.org/html/1277/127752022003/>. Acesso dia 07 Mar. 2018.

RABELO, L. R.; OLIVEIRA, D. L. Percepções de enfermeiras obstétricas sobre sua competência na atenção ao parto normal hospitalar. Rev. Esc Enferm USP 2010; 44(1):213-20. Disponível em:

<http://www.scielo.br/pdf/reeusp/v44n1/a30v44n1.pdf>. Acesso em: 23 Jan. 2018.

RAMOS, W. M. A. et al. Contribuição da enfermeira obstétrica nas boas práticas da assistência ao parto e nascimento. Rev Fund Care Online. 2018 jan./mar.; 10(1):173-179. Disponível em:

<http://pesquisa.bvsalud.org/portal/resource/pt/bde-32254>. Acesso em: 27 Fev. 2018.

REIS, T. R. et al. Enfermagem obstétrica: contribuições às metas dos Objetivos de Desenvolvimento do Milênio. Rev Gaúcha Enferm. 2015;36(esp): 94-101. Disponível em: <http://www.scielo.br/pdf/rgenf/v36nspe/01026933-rgenf-36-spe-0094.pdf>. Acesso em: 22 Jan. 2018.

SANTOS, R. A. A.; MELO, M. C. P.; CRUZ, D. D.Trajetória de humanização do parto no Brasil a partir de uma revisão integrativa de literatura. Caderno de Cultura e Ciência, Ano IX, v.13, n.2, Mar, 2015. Disponível em: <http://periodicos.urca.br/ojs/index.php/cadernos/article/view/838/pdf_1>. Acesso em: 08 Mar. 2018.

SILVA, T. C. et al. Práticas de atenção ao parto e nascimento: uma revisão integrativa. Revista de Enfermagem do Centro-Oeste Mineiro. 2017;7:e1294. Disponível em: <http://pesquisa.bvsalud.org/portal/resource/pt/bde30815>. Acesso em: 27 Fev. 2018.

VIEIRA, M. J. O. et al. Assistência de enfermagem obstétrica baseada em boas práticas: do acolhimento ao parto. Rev. Eletr. Enf. [Internet]. 2016. Disponível em: <https://revistas.ufg.br/fen/article/view/36714/21669>. Acesso em: 27 Fev. 2018. 